\title{
Engaging Audiences through a Participatory Design Approach with the Interactive Music Installation 'SoundField'
}

\author{
Alexander Deweppe \\ iMinds-SMIT-VUB \\ Pleinlaan 9, 1050 Brussel \\ Alexander.Deweppe@vub.ac.be
}

\author{
Nuno Diniz \\ IPEM-UGent \\ Blandijnberg 2, 9000 Gent \\ nuno.diniz@gmail.com
}

\author{
Pieter Coussement \\ IPEM-UGent \\ Blandijnberg 2, 9000 Gent \\ pieter.coussement@telenet.be
}

\author{
Marc Leman \\ IPEM-UGent \\ Blandijnberg 2, 9000 Gent \\ Marc.Leman@UGent.be
}

\begin{abstract}
A rise in the availability of digital tools and interfaces that enable interactivity in a cultural context has kindled the interest from scientific research and creative industries alike. These technologies interface art in such a way that they create new collaborative and interactive possibilities for audience participation and artistic expression. A somewhat hesitant attitude of the cultural sector aside, the main adoption issue can be described as a dichotomy between the benefit of increased artistic opportunities, and the problems with readiness, eagerness and domestication from the part of the audiences.
\end{abstract}

\begin{abstract}
A set of exploratory studies was conducted to define the main problems that permeate the hesitant adoption process. A questionnaire and two interview studies (set up around actual interactive art installations within a museum context) elucidate the issues with technologically enhanced participation in receptive cultural activities. As a result of these preparatory studies, an $\mathrm{HCl}$-based Living Lab methodology was advocated and applied to an open-ended interactive system to attempt overcoming some of the issues stated in the field studies. The adoption of an iterative, use case and scenario-based, user-centered design method is consequently presented in the description of the resulting 'SoundField'-project.
\end{abstract}

In the result section, the rendition of several use cases -created for six different artistic practicesis described. The merits of engaging the audience within the development process are discussed, as the participatory design strategy and the implementation of user-informed design decisions prove to be instrumental in defining viable application domains for interactive artistic technologies. Finally, aside from being an efficient probing methodology for artistic objectives, the participatory strategy helps bridging the gap between the developers' abilities and users' aspirations, establishing better acceptance of the technology among audiences.

\section{Keywords: Participatory Design; Embodied Music Mediation Technology; Collaborative Music Interfaces;} Interactive Art Installations; Technology Adoption.

\section{INTRODUCTION}

Recent decades saw a significant increase of new digital technologies that enable and support cultural interaction and artistic expression (Paradiso and O'Modhrain 2003). In scientific as well as artistic research and creative industries, a growing interest for these technologies can be attested and efforts on the development of collaborative and interactive interfaces are being done. These technologies have the potential of interfacing art in such a way that they enable new possibilities for audience interaction and artistic expression for performers and audiences alike (Miranda and Wanderley 2006).
Leading institutions of the cultural sector do not always take an unambiguous stance towards this relatively recent and innovative phenomenon and have an at best hesitant attitude towards collaborative and interactive artistic technologies. Aside from considerable additional investments required, recurrent reliability issues that to date pervade implementations with state-of-the-art technologies (Norman 2010) and the level of technical expertise required for the maintenance of such technologically enhanced interaction spaces can be mentioned among the chief reasons. Ensuing adoption issues are a result of the problematic relation with readiness, eagerness, adoption and domestication of the technology that 
impair the demonstrable interactive benefits of the technological advances and the increased artistic opportunities, despite the technological availability. Therefore, the underlying research question focuses on what aspects of the cultural sector, its institutions and its target audiences these acceptance and adoption problems are mainly related to.

The aim of the underlying studies is to identify to what extent the adoption is hindered by solely technological restrictions, as well as to identify other factors. In three preliminary studies, attempts are made to define issues that permeate the hesitant adoption process. First, a questionnaire is presented that was conducted among a predominantly young and technology-skilled audience with active cultural profiles, probing technological literacy and attitudes towards technological participation in cultural receptive activities. Then, two in situ interview studies are discussed, which were set up around actual interactive art installations. In these studies, the methodological problems with acquiring relevant information from potential end-users will be illustrated. As a result of these studies, an $\mathrm{HCl}$ based methodology is advocated to solve some of the issues stated in the empirical field studies. The adoption of an iterative and participatory design methodology applied to the cultural sector is then presented in the description of the resulting the 'SoundField'-project.

\section{THEORETICAL BACKGROUND}

Collaborative and interactive interfaces for musical expression (Fels 2005) are a relatively novel artistic phenomenon brought about by the merging of sufficiently powerful computers on the one hand, and the commercial availability of affordable and reliable tracking technologies (e.g. sensors and motion capture devices) on the other. In cooperation with these technological mediators, the human body can function as an augmented instrument, expanding the possibilities of musical interaction and experience within a technologically extended environment (Vines et al. 2006). In accordance with the paradigm of embodied music cognition, the mediation of movement can thus be considered an extension of the body into the artistic realm, increasing the operational and aesthetic range of performer and/or public (Leman 2008).

Both the scientific community and the creative sector strive to allow the embodied mind to express its musical intentions through the use of new technologies for interactive sound creation. The goal is to build embodied music controllers that are unbound by the fixed interaction principles prescribed by more traditional interfaces (i.e. 'conventional' music instruments). This humanmachine interaction paradigm (Godoy and Leman 2009 ) is grounded in the idea that action and perception are tightly coupled and embedded within the same sonic action-reaction-cycle, in which the repertoire of gestures and actions is referred to as the human action-oriented ontology (Leman 2008). When an engaging interactive technology allows artistic affordances (Jones 2003) to a participant, theoretically, a communicative process in which an interactive augmented reality environment takes on the role of social agent (Leman 2008) can occur, and hence meaningful musical interactions between user and device can exist. Consequently, new opportunities for social interaction can also come about, as the participants of this musical communication process are no longer tied to stationary controls or typical interfaces.

Yet, in meeting the aesthetic and functional requirements of a given artistic realization, interactive technologies tend to fall short. In scientific reviews, collaborative interfaces are frequently presented as highly technological music toys or games (Fels, 2005), rather than artistic interactive instruments or installations in their own right. Additionally, quite often, the artistic purpose is difficult to assess by the audience when confronted with interactive music installations, and the given affordances are opaque, making the technology difficult to read, and experiences with it more difficult to discern, understand and appreciate.

From the part of cultural institutions, interactive installations are considered difficult to operate and implement, as a number of complex and intricate technologies lie at the basis (Diniz et al. 2011). From the part of the audiences, it can be noted that a substantial level of media literacy (Livingstone 2004) and advanced technology skills (Hobbs 1998) are desirable to access and grasp the aesthetic possibilities of this art form. Consequently, the reservations in the adoption process of the described technology that prevail in large segments of the cultural sector can be easily understood. In an attempt to reverse this tendency, research institutes have started to advocate new approaches that draw on methodologies borrowed from $\mathrm{HCl}$ (Wanderley and Orio 2002) to make embodied music mediation technologies more practicable in pre-existing cultural contexts. Experiments with advanced and flexible setups have been conducted in artistic incubators (Paradiso 1999) and within cultural institutions (Freeman 2008) to further the understanding of these technologies and to facilitate adoption and domestication of interactive music installations in the sector (Ferguson and Wanderley 2010).

\section{EMPIRICAL STUDIES}

\section{1 'What Moves You'}

Various national and cross-national studies on culture and innovation investigate this changed reality of cultural and creative economy brought 
about by the exponential rise of new media applications at the turn of the century. Pivotal in these studies are the diversification of the actors in the concentric model of the cultural industries (Throsby 2008) and the definition of challenges and opportunities for the creative economy in various regions (Power 2009). Central topics to this line of research are the various trends and perspectives of new media adoption that can be discerned. Traditionally, studies focused on (inter)national and regional differences in adolescents' media use (Roe 2000), on distinguishing differences between old and new media usage for young populations (Johnsson-Smaragdi et al. 1998), or on specificities of media literacy and audio-visual media consumption with specific age groups (Adoni and Nossek 2001). More recently, also gender divides in ICT attitudes (Broos 2005) and other psychological factors that influence ICT adoption (Broos and Roe 2006) are being considered, pointing out that the digital divide is more than a merely generational phenomenon.

New media attitudes are known to differ significantly by gender, age, family dynamics, geographical factors, social status, economic position and education levels, yet general tendencies are very hard to convey by probing specific populations (Lievens and Waege 2011). Accordingly, the systematic exclusion of variables can more easily result in a realistic assessment of different profiles of new media adoption (Schuurman et al. 2011). Moreover, when considering the impact of and attitudes towards new media in the different segments of the culturalcreative economy, a substantial amount of different viewpoints (sociology, psychology, economy and marketing, human computer interaction and informatics, etc.) are required to establish a nuanced perspective. Namely personality assessments, musical identities (Rentfrow and Gossling 2003) and in a broader sense cultural profiles (Roose 2008) of the stakeholders are key elements to gain insight into the complex nature of a rapidly changing cultural sector.

In preparation of a development of interactive music tools based on the paradigm of embodied music cognition (Leman 2008), creative strategies for the implementation of gestural music mediation technologies were required. To fit prospective users' needs, information about the future participants, the context of use and purpose they serve is required. For this purpose, a survey of the Flanders cultural landscape was made. The rationale was to organise a panel discussion between the various stakeholders, in which cultural institutions, creative industries, research departments and prospective end-users could collaboratively assess opportunities and their viability of embodied music mediation technologies within the cultural sector. With respect to the endusers, this information was acquired by means of a questionnaire, entitled 'what moves you', in which relevant viewpoints concerning embodied music mediation technology were probed. The purpose was to get an overview of a young, technologysavvy portion of the stakeholders. Moreover, the questionnaire aimed at gaining insight into relevant convictions for the development of such interactive tools. Finally, the survey wanted to identify possibilities for the efficient and effective embedding of the technology. In parallel with this survey of potential end-users' cultural profiles, artistic education, cultural background and new media behavior, an attempt was done to create an annotated pool of potential prospective users among culturally active audiences.

The WMY-questionnaire collected information covering a total of seven topics: demographic information, personality traits, background and education in music and dance, cultural and musical preferences, movement and dance as a response to music, dance behavior and preferences, acquaintance, usage, literacy and attitudes towards new technologies. The survey was conducted between late 2009 to spring 2011. In this period over five hundred respondents participated in the questionnaire. Though quite specific in nature and with an explicitly exploratory purpose (i.e. attuned to embodiment and parameters relevant to the development of embodied music mediation technologies), the results of this questionnaire overall followed some of the large basic tendencies of authoritative participation (Lievens and Waege 2011) and media acquisition studies (Schuurman et al. 2011) in Flanders.

The collected data showed a slight overrepresentation of female respondents, and the age group under 35 years old made up $85 \%$ of the total number of respondents, of which $67 \%$ were aged between 16 and 25 . The respondents were overall highly educated and over $60 \%$ of the respondents had received formal music education for one or more years. Nearly all of the respondents engaged in cultural activities at least once a month. Concerning the attested profiles in new media technologies and the respondents' attitudes towards innovation, about $10 \%$ of the respondents considered themselves to be lead-users, whereas about a third of the respondents took an indifferent or negative stance towards new technologies. In terms of technology acquisition, less than $10 \%$ were early adopters and about half of the respondents said to generally wait quite long before they acquired new technologies.

Concerning technologically mediated audience interaction in artistic contexts, results suggest that only $10 \%$ is sincerely enthusiastic about the prospect of technology-enhanced participation. About $40 \%$ of the participants took a hesitant stance (i.e. not opposed to the idea, but with reservations concerning the implied new role of audience participation). Close to half the 
respondents had an uninterested or downright negative attitude towards it. Less than a quarter $(23.6 \%)$ of the respondents was enthusiastic about the prospect of being actively involved in influencing or triggering sonic or visual content.

Summarizing, a predominantly young sample of highly educated culture- enthusiasts, most of which had received some form of artistic training and most of which had demonstrable media skills, were asked about their opinions concerning technological advances in a receptive cultural context and the possibility of technology-assisted participation. The majority of respondents expressed concerns or was overtly unenthusiastic about this prospect.

\section{2 'Heart as an Ocean' and 'Lament'}

As the previous study ascertained some general misgivings concerning technologically enhanced participation, two smaller exploratory in situ tests were conducted, focusing on the in-depth and reallife experience of culture-enthusiasts when interacting with artistic installations. These tests were conducted with two different interactive installations, namely 'Heart as an Ocean' and 'Lament' (Deweppe et al. 2011).

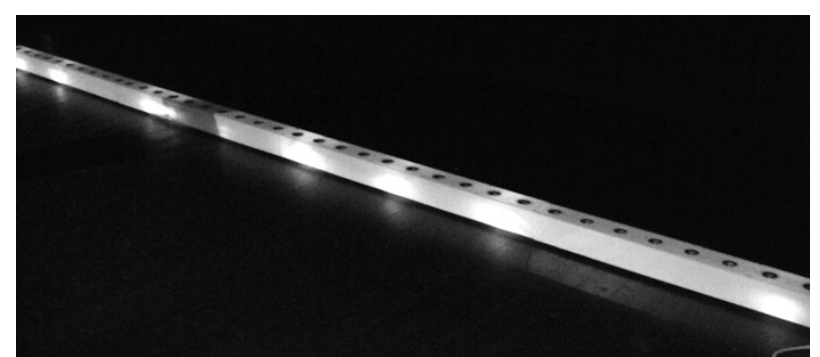

Fig. 1: 'Heart as an Ocean', an action-perception coupling art installation using biometric feedback

'Heart as an Ocean' (Fig. 1) was designed to reflect the state of mind of any person who interacted with the installation as an individually responsive installation. A semi-fixed interview was prepared to evaluate the users' experiences within the context of the artwork, immediately after interaction. In addition to standard socio-demographic questions and information concerning cultural background, some questions relevant to the participants' experience dealt with their comprehension, the perceived purpose, the personal evaluation, quality of operation and sensory perception of the installation's output. Since there was no definable task, no usability metrics or heuristics were applied in this context.

The majority of visitors did not want to interact with the installation while being observed. Then, even though most of the actual participants were willing to partake in the interviews after experiencing the installation, the results were very inconclusive. The majority of the respondents claimed to have understood the basic operation of the installation and to have enjoyed the experience. However, the responses of over forty participants that were interviewed were so diverse, that no classifications of their responses could be made and no further analysis could be performed. This study adequately demonstrated how difficult it was to successfully gather user feedback when dealing with a finished art piece and within a pre-existing art context.

The second installation, 'Lament' (Fig. 2), was not designed to be experienced individually, but was intended to provoke participants to actively and collaboratively experience a sonic environment. The public could easily connect to 'Lament' because of its low threshold and iconic visual identity, which elicited interaction.

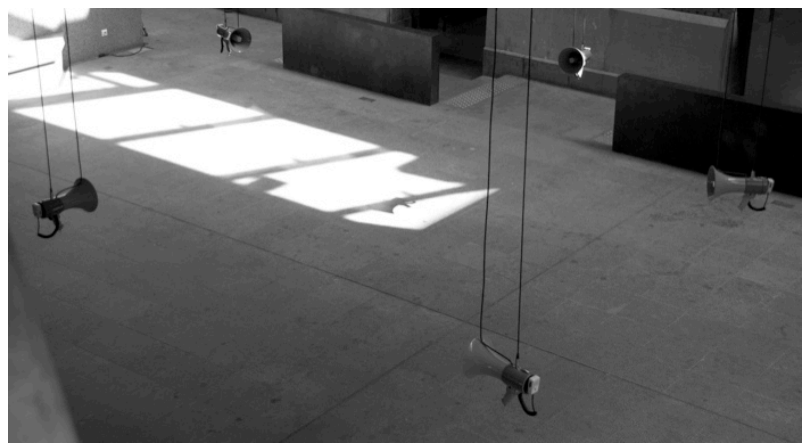

Fig. 2: the 'Lament' installation, presented at music centre Bijloke, Ghent

Next to the installation, a computer terminal enabled the public to report on their appraisal of the artwork and to evaluate their experience by means of computer-based questionnaire. A first set of questions pertained to the participants' awareness of the installation, to the functional and aesthetic properties of the art- work, to the responsiveness, to the interactive nature of the art-piece and to the assessment of the interaction. The second part probed familiarity with new media and installation art, alongside standard socio-demographic questions.

During the week 'Lament' was exhibited, approximately 4000 visitors attended the music center where it was shown. Conversely, no more than fifty attendees filled out the questionnaire. This once more exemplifies the difficulty of gathering feedback on location and getting participants to comment on their experiences. Considering the outcome of these pilot studies, no vital scientific information was gained from either of the two events. Methodologically, however, both studies had significant merits into research on artistic interactive applications.

In the first instance, concise and precise information was expected. Conversely, the experience-based feedback that was gathered was elaborate and rich. With the aim of reporting back to the artist on the experiences with the interactive installation of the participants, this was valuable feedback. Alternately, for making broad 
assessments on the global experience of participants interacting with the installation, it proved to be ill suited.

In the second instance, the formalised online questionnaire left less to chance, yet it was the response ratio (i.e. approximately $2 \%$ ), which presented the main issue here. Even though well advertised, only a very limited number of dedicated culture-enthusiasts participated in the questionnaire. The majority of visitors to the festival where 'Lament' was presented could not be reached using this strategy. Moreover, from the limited number of questionnaires that were completed, the questions pertaining to additions and changes that could be made to the setup, remained practically unanswered.

In addition to the earlier findings of the 'what moves you'-questionnaire, namely that, inconsiderate of age, even a culturally highly active audience is not overly eager to interact with unfamiliar interactive interfaces, this illustrates the difficulty of engaging target-audiences in artistic research. In both instances, it was clear that merely exhibiting quasifinished art pieces in a museum context was not an efficient way of (1) conducting research on interactivity, (2) attracting new audiences for this type of artistic expression or of (3) further developing interface-based artistic installations. Additionally, the above experiments illustrate that, concerning interrogation of culture-enthusiasts, interview and questionnaire strategies are complementary. Both are indispensable to get parts of the feedback that is vital to inspire further development, but the inquiry form is key in view of required information.

\section{RESULTS}

Based on the combined experiences of the previous studies, the decision was made to collaborate with end-users in a creative environment and within an artistic context for the development of an installation, to tackle the issues described above. For this purpose, the 'SoundField'-platform was developed, an interactive, virtual and immersive environment, that makes physical interaction with visual and sonic content possible. It was essentially an open-ended black box artistic installation based on a flexible, multi-modal enabling java-framework, which functions as a testbed for different modes of sonic, visual an social interaction, usability (and interface) evaluation and use case development.

This research platform was presented in a genuine cultural context and encompassed a living lab methodology and participatory design process for the investigation of $\mathrm{HCl}$-based functional parameters. It could accommodate a variable number of participants and it presented a space in which image and sound could be created, influenced, manipulated or explored by means of virtual object mediation in an augmented space
(Fig. 3). Eventually, the project targeted end-user and audience customization and acceptance.

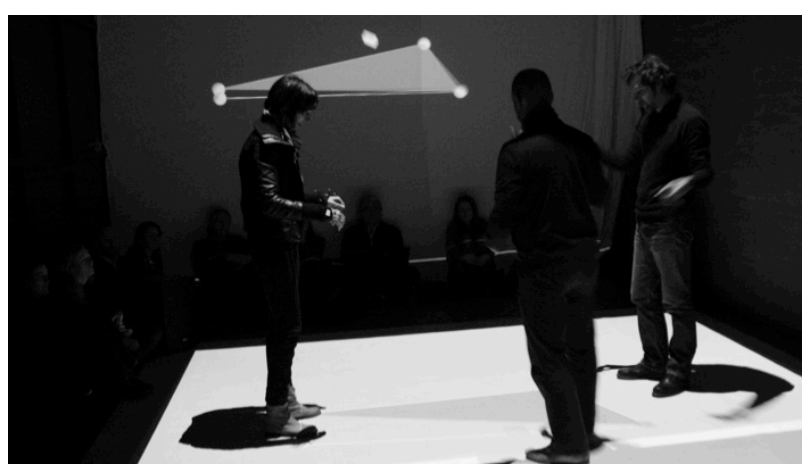

Fig. 3. Three users interacting with the demo of the 'SoundField'-platform

\subsection{Technical setup and interaction principles}

The setup consists of a set of four projectors, a ten infrared camera array that tracks rigid body movement and a quadrophonic speaker system. The cameras constantly track the position of the participants, enabling the user to experience corporeal control over the sonic and visual environment, and creating a forum for the exploration of new modes of creative sound and image interaction. As the subjects move through the room, they can effect change in the projected images and the produced sound. The information from camera- array is gathered, sent to the javaframework and parsed to Java-3D or Max/MSP/Jitter in combination with Ableton Live for sonic and visual output.

The setup is, both in terms of content and interaction, essentially open-ended. In collaboration with the users, we did reflect on the framework, on its purpose and on its (possible) contents, on improving ergonomics, etc. Using a multi-view orthographic projection and a combination of infrared motion tracking and customizable userinterface devices, 'SoundField' provided real-world access to objects existing in the projected virtual reality.

The different levels of interactivity were defined by increasing spatial dimensions (loosely based on Kandinsky's classes of form): from a single point in space, over to a line, which connects two points, then to a surface (plane, where a minimum of three points were interconnected). Each of these dimensions corresponded to an equivalent aural dimension, where sounds gained in complexity throughout the increased dimensionality. Additionally, the dimensions were linked to social interaction potential that are accordingly represented in the various accessible levels of the installation. The larger the number of participants and the more social interaction, the fuller the interaction with the installation became. As a consequence, the installation was devised in such a way that the higher interaction levels of the 
system could only be accessed when participants interacted and collaborated in it. As such, 'SoundField' functioned as a catalyst for social interaction, more than merely a collaborative controller for sound and image manipulation.

\subsection{Design strategy}

Over an active period of four months, several fully functional implementations of the technology were developed according to a formative user-centered evaluation cycle (Gabbard et al. 2002). After a short introduction and demonstration of the platform, a small group of users were asked to interact with an abstract demo-version. During these interactive demo-sessions, participants were invited to describe their actions, much in the way that an interaction design session is conducted (Barrass 2008) and consecutively were asked questions about their performance. They were also encouraged to ask questions, to improve their understanding of the installation.

After the demo-session, people were asked to participate in a focus group session that was conducted with a number of participants ranging from 5 to 20. Even though the main purpose of the demonstration was to show participants what the framework was capable of, it was also an intention of this session to record the spontaneous ideas and associations of the participants. Consequently, the artistic background of the coinciding workshops, the aspirations expressed by the participants, their questions, remarks, findings and feedback gave a clear direction to each one of the use cases. Furthermore, due to the experience the focus group participants had gathered during the interaction session, their expectations concerning clarity, ease of use, control and aesthetic properties could also be discussed in the focus group session.

In this manner, prior to the implementation process, the developers could gather a very thorough insight into what needed to be included in this iteration, within the scope of the possibilities inherent in the framework. Then, through rapid prototyping, the requested features were incorporated and presented to the same groups of users. Another run of exploratory tests and interaction sessions was conducted, after which the overall presentation, the specifically implemented features of the given use case and the experience of the users were recorded in a questionnaire. To be able to incorporate the developers' experiences in each of the consecutive use cases, the same procedure was applied throughout all iterations.

In summary, the participatory design and iterative development process described above was carried out throughout the entire duration of the project. Every use case featured (1) a demonstration, (2) an interrogation, (3) the implementation of the features, (4) an interaction session and (5) a questionnaire-based evaluation. Only for those use cases that were solely devised for demonstration purposes, this last step was not included.

\subsection{Use cases and iterations}

A number of demos were done between July 2010 and October 2010 with a representative and varied test audience. Here a laptop-based demonstrator of the eventual platform was presented as the developmental strategy was tested. During these sessions, ideas emerged that inspired either implementation strategies or interaction scenarios. From October 2010 to February 2011, in close collaboration with the Flanders "Youth and Music"program, the 'SoundField'-installation was presented to a broader audience at Destelheide Arts Education centre in Dworp, Belgium.

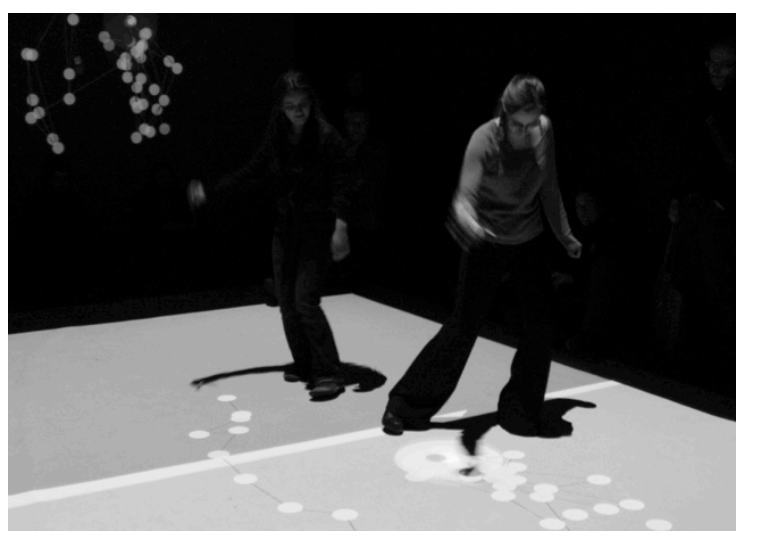

Fig. 4. Two users interacting with the 'SoundTracks'-use case

To be able to achieve good results by means of rapid prototyping, a number of predefined and preprogrammed elements (spheres, stings, membranes), selectable element-behaviors (fixed, semi- fixed or mobile) and scenarios (flexible combinations of the latter) were devised. Each time the platform was put to use for a specific artistic purpose and within a given cultural practice, the end-result would be defined as a use case.

In total, twenty-four use cases were created and six different types of use cases can be defined, namely (1) demonstration use cases, (2) sound manipulation use cases, (3) sonification tools, (4) choreography tools, (5) theatrical use cases and (6) movie manipulation tools (Fig. 4).

\section{RESULTS}

The conclusions that are applicable to all of the use cases are limited, given the broad range of application domains. Still, some general findings can be presented. First of all, the project exemplifies the large benefits of a user-centered design strategy in the development of artistic technologies and user-informed decision-making in the implementation of augmented reality environments for the cultural sector. It proved to be 
an efficient probing methodology as the participatory design strategy helped bridging the gap between developers' capabilities and ambitions on the one hand, and users' aspirations on the other.

Methodologically, this project was entirely based around participatory and iterative design. It relied heavily on the indispensable and active incorporation of users, participants and artists over the course of the entire project. In combination with the in situ-work in an artistic incubator, it created the opportunity for the developers to get a clear idea on goal assessment, which in turn enabled the creation of user-molded artistic tools. The iterative development process allowed for user-informed design adjustments and finally, the evaluation method created an opportunity for analyzing the quality of the presented developments (Fig. 5). Moreover, it offered a realistic alternative for taskbased usability and efficiency metrics. The artistic adaptation of standardised human computerinteraction heuristics for gesture-based interfaces also made a practicable contribution to cultural quality of experience measurement.

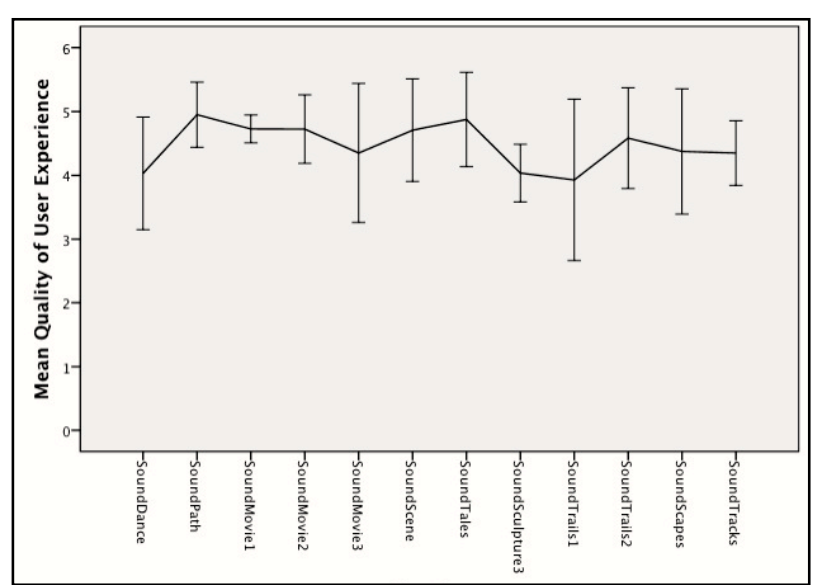

Fig. 5. Post-interaction user evaluation of a selected sample of use cases

Through the fruitful collaboration of developers and manageable numbers of participants, over twenty fully functional use cases could be developed in a period of just over four months. This facilitated the swift execution of ideas and a broad range of different cultural practices and conceivable applications to be explored.

Finally, this project was a testbed for technological development, for interactivity in art and for goal assessment and technology adoption within the cultural sector. Its main contribution is that, albeit exploratory, a number of conceivable uses for this type of technology have been dealt with. Solely based on this project, it is very difficult to assess whether or not this is a viable means of establishing technology acceptance in the cultural sector as such, but the active incorporation of the target-audience in the designing of the interactive tools undoubtedly has demonstrable merits to overcome some of the issues stated earlier (e.g. literacy, skills and acceptance).

\section{DISCUSSION}

The impact digitization on all aspects of everyday life has become omnipresent over the last decades. Confronted with that modern-day digitised society, the cultural sector by and large struggles with implementation of state-of-the-art technologies, as it presents threats as well as opportunities. Initiatives to adapt to this changed reality are in existence, but today's cultural sector and its audience have a hard time dealing with this fastchanging ICT-reality and its impact on the sector.

Established cultural institutions may be somewhat reluctant towards technology-informed change, additionally, their dedicated target-audiences show a tendency to take a similar hesitant stance. So, for the time being, going with the trend appears to be ill advised and gratuitous (Power 2009).

On the other hand, in due course, there are plenty of opportunities and promising circumstances to implement technologies in the cultural sector. First, the cultural sector has more opportunity for experimentation with innovative technologies than other sectors (Pratt 2005). Accordingly, as it is to a lesser extent subjected to "single point of launch"strategies than is the case with more conventional ICT-innovations, there is more room for openmindedness towards new technologies, without the explicit and immediate risk of failure. And finally, as culturally engaged audiences tend to have a sense of institution-loyalty, aspects of competitiveness have a less firm grasp on the cultural sector than on more industrialised branches of the creative industries.

Collaboratively constructed installations, designed along the lines of the presented participatory design methodology and drawing on the desires and expectations of the audience, may eventually prove to be quite useful in improving the participants' understanding, enjoyment and overall quality of experience when interacting with cultural contents. Bearing these findings in mind and with end-user research in the cultural sector in the early stages of development, we are still faced with vast challenges, but also with great opportunities.

A lot of work remains to be done on a societal, a technological and on an institutional level. Still, similar initiatives may eventually be the way, albeit one small but valuable step at a time, towards user understanding and user-acceptance of embodied music mediation tools.

\section{REFERENCES}

Adoni, H. and Nossek, H. (2001) The new media consumers: Media convergence and the displacement effect. Communications 26(1), 59-84. 
Barrass, S. (2008) Creative practice-based research in interaction design. Computers in Entertainment (CIE) 6(3), 1-17.

Broos, A. (2005) Gender and information and communication technologies (ICT) anxiety: male self-assurance and female hesitation. CyberPsychology \& Behavior 8(1), 21-31.

Broos, A. and Roe, K. (2006) The digital divide in the playstation generation: Self-efficacy, locus of control and ICT adoption among adolescents. Poetics 34(4-5), 306-317.

Deweppe, A., Diniz, N., Coussement, P. and Leman, M. (2011) A methodological framework for the development and evaluation of user-centered art installations. Journal of Interdisciplinary Music Studies 5(1), 19-39.

Diniz, N., Coussement, P., Deweppe, A., Demey, M. and Leman, M. (2012) An embodied music cognition approach to develop multilevel interactive sonification. Journal of Multimodal User Interfaces 5(3-4), 211-219.

Fels, S. (2004) Designing for intimacy: Creating new interfaces for musical expression. Proceedings of the IEEE, 92(4), 672-685.

Ferguson, S. and Wanderley, M.M. (2010) The McGill Digital Orchestra: An Interdisciplinary Project on Digital Musical Instruments. Journal of Interdisciplinary Music Studies 4(2), 37-54.

Freeman, J. (2008) Extreme sight-reading, mediated expression, and audience participation: Real- time music notation in live performance. Computer Music Journal 32(3), 25-41.

Gabbard, J.L. Hix, D. and Swan, J.E. (2002) Usercentered design and evaluation of virtual environments. Computer Graphics and Applications, IEEE 19(6), 51-59.

Godoy, R.I. and Leman, M. (2009) Musical Gestures: Sound, Movement, and Meaning. Routledge, London.

Hobbs, R. (1998) The seven great debates in the media literacy movement. Journal of Communication 48(1), 16-32.

Johnsson-Smaragdi, U., d'Haenens, L., Krotz, F. and Hasebrink, U. (1998) Patterns of old and new media use among young people in Flanders, Germany and Sweden. European Journal of Communication 13(4), 479-501.

Jones, K.S. (2003) What is an affordance. Ecological psychology 15(2), 107-114.

Leman, M. (2008) Embodied Music: Cognition and Mediation Technology, The MIT Press.

Lievens, J. and Waege, H. (2011) Participatie in Vlaanderen 1; Basisgegevens van de participatiesurvey 2009. Acco, Leuven.
Livingstone, S. (2004) Media Literacy and the Challenge of New Information and Communication Technologies. The Communication Review 7(1), 314.

Miranda, E.R. and Wanderley, M.M. (2006) New digital musical instruments: control and interaction beyond the keyboard, Middleton, Wis.: A-R Editions.

Norman, D. (2010) Gestural Interfaces: A Step Backward In Usability Design. Interactions 17(5), 46-49.

Paradiso, J.A. (1999) The brain opera technology: New instruments and gestural sensors for musical interaction and performance. Journal of New Music Research 28(2), 130-149.

Paradiso, J.A. and O'Modhrain, S. (2003) Current Trends in Electronic Music Interfaces. Guest Editors' Introduction. Journal of New Music Research, 32(4), 345-349.

Power, D. (2009) Culture, creativity and experience in Nordic and Scandinavian cultural policy. International Journal of Cultural Policy 15(4), 445460.

Pratt, A.C. (2005) Cultural industries and public policy. International Journal of Cultural Policy 11(1), 31-44.

Rentfrow, P.J. and Gosling, S.D. (2003) The do re mi's of everyday life: the structure and personality correlates of music preferences. Journal of personality and social psychology 84(6), 12361256.

Roe, K. (2000) Adolescents' media use: a European view. Journal of Adolescent Health 27(2), 15-21.

Roose, H. (2008) Many-Voiced or Unisono. Acta Sociologica 51(3), 237--253.

Schuurman, D., Courtois, C., De Marez, L. (2011) New media adoption and usage among Flemish youngsters. Telematics and Informatics 28(2), 7785.

Throsby, D. (2008) The concentric circles model of the cultural industries. Cultural Trends 17(3) 147164.

Vines, B.W., Krumhansl, C.L., Wanderley, M.M. and Levitin, D.J. (2006) Cross-modal interactions in the perception of musical performance. Cognition, 101(1), 80-113.

Wanderley, M.M. and Orio, N. (2002) Evaluation of input devices for musical expression: Borrowing tools from HCl. Computer Music Journal 26(3), 6276. 